\title{
Tracking of Voltage Flicker in Power Distribution Systems: A Local Polynomial Modelling Approach
}

\author{
Z. G. Zhang and S. C. Chan \\ Department of Electrical and Electronic Engineering \\ The University of Hong Kong, Pokfulam Road, Hong Kong, China \\ \{zgzhang; scchan\}@eee.hku.hk
}

\begin{abstract}
This paper proposes a local polynomial modelling (LPM) approach for voltage flicker tracking problem in power systems. The estimation of voltage envelope can be regarded as the identification of a time-varying linear model (TVLM). The proposed LPM approach models the time-varying coefficients of the TVLM locally by polynomials, which can be estimated by least-squares estimation with a kernel having a certain bandwidth. A data-driven variable bandwidth selection (VBS) method is then proposed to estimate the optimal variable bandwidth for minimizing the mean squared error (MSE). Online implementation of the LPM method for continuous tracking is also developed. The usefulness of the proposed approach is demonstrated by its improved performance over other conventional tracking methods in a variety of simulation scenarios and for voltage flicker tracking in a simulated power distribution system.
\end{abstract}

\section{INTRODUCTION}

In power distribution systems, voltage flicker or fluctuation is often produced by high electricity load alternations, such as arc furnace operations and resistance wielding, and it will cause serious quality problems to the power systems and consumers [1]-[5]. Accurate envelope tracking of the voltage measurements is therefore important not only for evaluating the flicker level, but also for compensating the flicker and regulating the voltage. Usually, the measured voltage $v(t)$ can be modelled as a sinusoidal waveform with time-varying amplitudes as follows [5]:

$$
\begin{aligned}
v(t) & =A(t) \sin (\omega t+\phi)+e_{v}(t) \\
& =A(t)[\sin \omega t \cos \phi+\cos \omega t \sin \phi]+e_{v}(t) \\
& =[\sin \omega t, \cos \omega t]\left[\begin{array}{l}
A(t) \cos \phi \\
A(t) \sin \phi
\end{array}\right]+e_{v}(t) \\
& =\Omega^{T}(t) \Phi(t)+e_{v}(t),
\end{aligned}
$$

where $A(t)$ is the voltage amplitude, $\omega$ is the supply angular frequency, $\phi$ is the phase angle, and $e_{v}(t)$ is the additive measurement noise. The voltage envelope is obtained as the $L_{2}$-norm of the estimated $\Phi(t)$.

The voltage model in (1) can also be viewed as timevarying linear model (TVLM), which has the form

This study was partially supported by the University of Hong Kong CRCG Small Project Funding.

$$
y(t)=\sum_{k=1}^{L} a(k, t) x(k, t)+\sigma(t) \varepsilon(t),
$$

or in the matrix form

$$
y(t)=\boldsymbol{a}^{T}(t) \boldsymbol{x}(t)+\sigma(t) \varepsilon(t) .
$$

In the TVLM of (2) and (3), $\boldsymbol{a}(t)=[a(1, t), a(2, t), \cdots, a(L, t)]^{T}$ is the time-varying coefficient vector of the system, which is assumed to be a function of discrete time instant $t, L$ is the order of the system, $\sigma^{2}\left(t_{0}\right)$ is the conditional variance of additive noise at $t=t_{0}$, and $\varepsilon(t)$ is an additive noise, which is frequently modelled as a zero mean white Gaussian process with unit variance. By comparing (3) with (1), we can see that, in the voltage flicker tracking problem, $v(t)$ is the measured output, $\Omega(t)$ is the known input, $\Phi(t)$ is the time-varying coefficient, and the system order $L$ is equal to 2 .

The TVLM is a simple and efficient model to characterize a dynamic discrete-time system with time-varying behaviors [6]. Given a dynamic system with known input and output, it is crucial to accurately identify the underlying dynamics of the system for predicting future measurements and detecting system variations. In the case of power distribution system, voltage flickers may cause serious power quality problems and they need to be estimated accurately. Generally, two classes of TVAR identification methods are commonly used in practice [6], and they are 1) adaptive filtering [7] and Kalman filtering (KF) [8]; 2) basis expansion modelling (BEM) [9]. Although these methods usually offer efficient implementation and different tradeoffs between performance and complexity, their performances are often dependent on prior information and model parameters [6]. Therefore, the estimation accuracy of these methods is sometimes limited when prior knowledge is vague or parameters are incorrectly specified.

In [13] and [14], a local polynomial modelling (LPM) approach is proposed to estimate the time-varying coefficients of TVLM's for tracking voltage flicker in power distribution system. The LPM technique is originally a flexible and efficient nonparametric approach in statistics [10] and it has been widely applied in data smoothing, derivative estimation, density estimation, etc [10]-[12]. To deal with the TVLM's, the proposed LPM method models each element of the timevarying coefficient vector locally by a set of polynomials with 
a kernel having a certain bandwidth. Consequently, the estimation of time-varying coefficients is reduced to the estimation of the local polynomial coefficients, which can be easily performed using the LS method.

The asymptotic behaviors of the proposed LPM estimator for TVLM have been studied in [13] and [14]. The asymptotic expressions of the estimation bias and variance show that both the bias and variance are functions of the bandwidth and there exists an optimal local bandwidth which minimizes the mean square error (MSE). While the analytical formulas of bias and variance are useful for theoretical work, they involve some quantities which may not be easily estimated in practice. Therefore, a "data-driven" variable bandwidth selection (VBS) scheme is proposed for selecting the local bandwidth in the LPM method. The basic idea of the "data-driven" scheme is to approximate the MSE of the LPM for a given bandwidth. Hence, an estimate of the optimal bandwidth can be obtained by minimizing the approximate MSE over a bandwidth set, which consists of a finite set of candidate bandwidth values. Following the classical approach in [10], a novel algorithm to approximate the MSE is proposed. It employs a "pilot" LPM of the system with a slightly higher polynomial order to estimate the bias and variance required in the approximated MSE. For tracking purposes, the optimal variable bandwidth can be updated in a recursive manner. Since this "pilot fit" also requires a pilot bandwidth, we propose to adopt the intersection of confidence intervals (ICI) method [11]-[15] for choosing this pilot bandwidth, because of its good performance and simple implementation. In the proposed LPM method with variable bandwidth selection (LPM-VBS), each coefficient in the coefficient vector is assigned a separate bandwidth, instead of using a global bandwidth for the whole coefficient vector. This facilitates the estimation and change detection of individual coefficient in the coefficient vector, which is very useful in fault detection and other applications. This study focuses on the online implementation of the LPMVBS method, and a number of practical issues are also discussed.

The performance of the LPM-VBS method was evaluated using various types of simulated TVLM's, and the results show that the proposed method yields more accurate estimates than several conventional TVLM identification methods in most testing scenarios. Lastly, the LPM-VBS method is applied to track the instantaneous voltage flicker in a simulated power distribution system. Simulation results show that the proposed LPM-VBS method has better performance than conventional methods in tracking the envelope of voltage flickers. These results suggest that the proposed LBM-VBS method can be a valuable tool in power system diagnostics and power quality monitoring.

The rest of the paper is organized as follows. In Section II, the local polynomial modelling for time-varying linear models is introduced. Section III is devoted to the development of the adaptive variable bandwidth selection method for LPM. Online implementation of the LPM-VBS method and several practical issues are discussed in Section IV. Simulation results and comparisons to conventional methods are presented in Section V. Finally, conclusions are drawn in Section VI.

\section{LOCAL POLYNOMIAL MODELLING OF TVLM}

In the proposed LPM method, the $k$-th coefficient $a(k, t)$ of the TVLM in (3) is modelled locally at time $t=t_{0}$ as a $p$-th order polynomial [10]:

$$
a(k, t) \approx \sum_{j=0}^{p} \frac{1}{j !} \alpha^{(j)}\left(k, t_{0}\right)\left(t_{i}-t_{0}\right)^{j},
$$

where $\alpha^{(j)}\left(k, t_{0}\right)$ are the associated polynomial coefficients. These polynomial coefficients can be estimated locally by maximum likelihood (ML) estimation. Since the additive noise is zero mean and white Gaussian distributed, maximizing the likelihood is equivalent to minimizing a locally weighted LS criterion between the observations and the desired local polynomials as follows:

$$
\min _{\beta} \sum_{i=1}^{n}\left[y_{i}-\sum_{k=1}^{L} \sum_{j=0}^{p} \beta^{(j)}\left(k, t_{0}\right)\left(t_{i}-t_{0}\right)^{j} x\left(k, t_{i}\right)\right]^{2} K_{h}\left(t_{i}-t_{0}\right),
$$

where $y_{i}=y\left(t_{i}\right), n$ is the data length, $\beta^{(j)}\left(k, t_{0}\right)=\alpha^{(j)}\left(k, t_{0}\right) / j !$, and $K_{h}\left(t_{i}-t_{0}\right)=\frac{1}{h} K\left(\frac{1}{h}\left(t_{i}-t_{0}\right)\right)$ is a weighting function which controls the bandwidth $h$ and hence the number of neighboring measurements around $t_{0}$ used to estimate $\beta^{(j)}\left(k, t_{0}\right)$. It can be seen that the weight function or kernel $K_{h}(\cdot)$ is obtained by scaling a basis kernel function $K(\cdot)$ in time by a factor of $h$. Note that $K(\cdot)$ can be selected as a one-sided kernel to meet the requirements of online tracking.

Next, we rewrite (5) more compactly in a matrix form as

$$
\min _{\boldsymbol{\beta}}\left(\boldsymbol{y}-\boldsymbol{X}\left(t_{0}\right) \boldsymbol{B}\left(t_{0}\right)\right)^{T} \boldsymbol{W}\left(t_{0}\right)\left(\boldsymbol{y}-\boldsymbol{X}\left(t_{0}\right) \boldsymbol{B}\left(t_{0}\right)\right),
$$

where $\boldsymbol{y}=\left[y_{1}, y_{2}, \cdots, y_{n}\right]^{T}, \boldsymbol{W}\left(t_{0}\right)=\operatorname{diag}\left\{K_{h}\left(t_{i}-t_{0}\right)\right\}$,

$\boldsymbol{X}\left(t_{0}\right)=\left(\begin{array}{cccc}\boldsymbol{x}^{T}\left(t_{1}\right) & \left(t_{1}-t_{0}\right) \boldsymbol{x}^{T}\left(t_{1}\right) & \cdots & \left(t_{1}-t_{0}\right)^{p} \boldsymbol{x}^{T}\left(t_{1}\right) \\ \boldsymbol{x}^{T}\left(t_{2}\right) & \left(t_{2}-t_{0}\right) \boldsymbol{x}^{T}\left(t_{2}\right) & \cdots & \left(t_{2}-t_{0}\right)^{p} \boldsymbol{x}^{T}\left(t_{2}\right) \\ \vdots & \vdots & \ddots & \vdots \\ \boldsymbol{x}^{T}\left(t_{n}\right) & \left(t_{n}-t_{0}\right) \boldsymbol{x}^{T}\left(t_{n}\right) & \cdots & \left(t_{n}-t_{0}\right)^{p} \boldsymbol{x}^{T}\left(t_{n}\right)\end{array}\right)$,

and $\quad \boldsymbol{B}\left(t_{0}\right)=\left\{\left[\boldsymbol{\beta}^{(0)}\left(t_{0}\right)\right]^{T}, \cdots,\left[\boldsymbol{\beta}^{(p)}\left(t_{0}\right)\right]^{T}\right\}^{T} \quad$ with

$\boldsymbol{\beta}^{(j)}\left(t_{0}\right)=\left[\beta^{(j)}\left(1, t_{0}\right), \cdots, \beta^{(j)}\left(L, t_{0}\right)\right]^{T}$. Note that $\boldsymbol{B}\left(t_{0}\right)$, $\boldsymbol{X}\left(t_{0}\right)$, and $\boldsymbol{W}\left(t_{0}\right)$ are all functions of $t_{0}$, but for notation simplicity we have dropped $t_{0}$ in subsequent text.

The LS solution to (6) is given by

$$
\hat{\boldsymbol{B}}=\left(\boldsymbol{X}^{T} \boldsymbol{W} \boldsymbol{X}\right)^{-1} \boldsymbol{X}^{T} \boldsymbol{W} \boldsymbol{y},
$$

and the $k$-th coefficient at time instant $t_{0}$ is obtained as $\hat{a}\left(k, t_{0}\right)=\hat{\alpha}^{(0)}\left(k, t_{0}\right)=\hat{\boldsymbol{B}}\left(k, t_{0}\right)$. By estimating $\hat{a}\left(k, t_{0}\right)$ at each time instant, we obtain a smooth function of the timevarying coefficients from the input $x$ and the noisy output $y$.

An important advantage of the proposed LPM method is that the bias and variance can be analytically derived, which paves the way to the solution of the key problem of automatic data-driven variable bandwidth selection. In [13] and [14], it has been proved that, as $n h \rightarrow \infty$ and $h \rightarrow 0$, the asymptotic bias and variance of the LS estimator in (7) are respectively expressed as 


$$
\begin{gathered}
\operatorname{Bias}(\hat{\boldsymbol{B}})=\left(\boldsymbol{H}^{-1} \boldsymbol{U}^{-1} \boldsymbol{u}\right) \otimes \boldsymbol{\beta}^{(p+1)} h^{p+1}\left\{1+o_{P}(1)\right\}, \\
\operatorname{Var}(\hat{\boldsymbol{B}})=\frac{\sigma^{2}\left(t_{0}\right)}{n f\left(t_{0}\right) h}\left(\boldsymbol{H}^{-1} \boldsymbol{U}^{-1} \boldsymbol{U}^{*} \boldsymbol{U}^{-1} \boldsymbol{H}^{-1}\right) \otimes \boldsymbol{R}_{X}^{-1}\left\{1+o_{P}(1)\right\},
\end{gathered}
$$

where $\boldsymbol{H}=\operatorname{diag}\left(1, h, \cdots, h^{p}\right), \quad\left(\boldsymbol{R}_{X}\right)_{1 \leq q, m \leq L}=r_{x, q, m}\left(t_{0}\right) \quad$ with $r_{x, q, m}\left(t_{0}\right)=E\left[x\left(q, t_{0}\right) x\left(m, t_{0}\right)\right] \quad, \quad(\boldsymbol{U})_{0 \leq j, l \leq p}=\mu_{j+l} \quad$ with $\mu_{\varsigma}=\int \tau^{\varsigma} K(\tau) d \tau,\left(\boldsymbol{U}^{*}\right)_{0 \leq j, l \leq p}=v_{j+l}$ with $v_{\varsigma}=\int \tau^{\varsigma} K^{2}(\tau) d \tau$, $\boldsymbol{u}=\left(\mu_{p+1}, \ldots, \mu_{2 p+1}\right)^{T}, \quad \boldsymbol{\beta}^{(p+1)}=\left[\beta^{(p+1)}\left(1, t_{0}\right), \cdots, \beta^{(p+1)}\left(L, t_{0}\right)\right]^{T}$ is the $(p+1)$-th derivative of $\beta, f(t)$ is the sampling density function at $t$.

It can be seen from (8) and (9) that, as $h$ increases, the squared bias will also increase while the variance will decrease. Hence, there exists a locally optimal bandwidth $h^{\text {opt }}\left(k, t_{0}\right)$ for estimating $\hat{\alpha}^{(j)}\left(k, t_{0}\right)$, which minimizes the MSE. However, as some of the quantities in (8) and (9) are difficult to be calculated directly, such as $\boldsymbol{\beta}^{(p+1)}$, the optimal bandwidth is difficult to be estimated accurately. We next introduce an empirical method to select the optimal bandwidth from a set of possible bandwidths.

\section{ADAPTIVE BANDWIDTH SELECTION FOR LPM}

Although the bias and variance cannot be directly computed because of the unknown quantities, good finite sample approximations of the bias and variance, can still be derived as in local polynomial regression [10]. Given a finite set of bandwidth parameters in an ascending order, say,

$$
\boldsymbol{H}=\left\{h_{j} \mid h_{1}<h_{2}<\cdots<h_{J}\right\} \text {, }
$$

where $J$ is the number of bandwidths, the empirical bandwidth selection method in [13] approximates the bias, variance, and MSE values of each bandwidth and determines an optimal bandwidth as the one that minimizes the approximated MSE.

For the estimation bias, which contains the unknown residual $\boldsymbol{r}=\boldsymbol{m}-\boldsymbol{X} \boldsymbol{B}$, can be estimated using a Taylor expansion with an order $p+p_{e x}$ :

$$
\boldsymbol{b}\left(\hat{\boldsymbol{B}}\left(t_{0}\right)\right)=\left(\boldsymbol{X}^{T} \boldsymbol{W} \boldsymbol{X}\right)^{-1} \boldsymbol{X}^{T} \boldsymbol{W} \boldsymbol{\tau},
$$

where $\boldsymbol{\tau}$ is an $n \times 1$ vector with $\sum_{v=1}^{p_{e x}} \sum_{k=1}^{L} \beta^{(p+v)}\left(k, t_{0}\right)\left(t_{i}-t_{0}\right)^{p+v} x\left(t_{i}-k\right)$ as its $i$-th $(i=1,2, \cdots, n)$ element, and the quantities $\beta^{(p+v)}\left(k, t_{0}\right)$ can be estimated by fitting a polynomial of degree $p+p_{e x}$. The basic idea is that the observations can be better fitted by a higher order polynomial, and hence the model provided by this higher order polynomial can be used to estimate the bias for a local polynomial model with lower order. Since the higher order model is still based on local polynomial modelling, we still need a bandwidth $h^{*}$ in this $\left(p+p_{e x}\right)$-th order LPM. This bandwidth $h^{*}$ is usually referred to as the "pilot bandwidth", which will be discussed later.

Next, suppose local homoscedasticity, the conditional variance can be estimated as:

$$
\boldsymbol{V}\left(\hat{\boldsymbol{B}}\left(t_{0}\right)\right)=\left(\boldsymbol{X}^{T} \boldsymbol{W} \boldsymbol{X}\right)^{-1} \boldsymbol{X}^{T} \boldsymbol{W}^{2} \boldsymbol{X}\left(\boldsymbol{X}^{T} \boldsymbol{W} \boldsymbol{X}\right)^{-1} \boldsymbol{\sigma}^{2}\left(t_{0}\right) .
$$

The noise variance $\sigma^{2}\left(t_{0}\right)$ is estimated as the normalized weighted residual sum of squares [10]:

$$
\hat{\sigma}^{2}\left(t_{0}\right)=\frac{\sum_{i=1}^{n}\left(y_{i}-\hat{y}_{i}\right)^{2} K_{h^{*}}\left(t_{i}-t_{0}\right)}{\operatorname{tr}\left\{\boldsymbol{W}^{*}-\boldsymbol{W}^{*} \boldsymbol{X}^{*}\left(\boldsymbol{X}^{* T} \boldsymbol{W}^{*} \boldsymbol{X}^{*}\right)^{-1} \boldsymbol{X}^{*^{T}} \boldsymbol{W}^{*}\right\}},
$$

where $X^{*}$ and $W^{*}$ are respectively the design matrix and weighting matrix in the $\left(p+p_{e x}\right)$-th order LPM using the pilot bandwidth $h^{*}$.

The MSE of the $v$-th entry of $\hat{\boldsymbol{B}}\left(t_{0}\right)$ at a given point $t_{0}$ is given by

$$
\operatorname{MSE}_{v}\left(\hat{\boldsymbol{B}}\left(t_{0}\right)\right)=b_{v}^{2}\left(\hat{\boldsymbol{B}}\left(t_{0}\right)\right)+V_{v}\left(\hat{\boldsymbol{B}}\left(t_{0}\right)\right),
$$

where $\hat{b}_{v}\left(\hat{\boldsymbol{B}}\left(t_{0}\right)\right)$ is the $v$-th element of $\boldsymbol{b}\left(\hat{\boldsymbol{B}}\left(t_{0}\right)\right)$ and $V_{v}\left(\hat{\boldsymbol{B}}\left(t_{0}\right)\right)$ is the $v$-th diagonal element of $\boldsymbol{V}\left(\hat{\boldsymbol{B}}\left(t_{0}\right)\right)$. Note that we only focus on the estimation of the coefficients $\hat{a}\left(k, t_{0}\right)=\hat{\alpha}^{(0)}\left(k, t_{0}\right)=\boldsymbol{B}\left(k, t_{0}\right)$ and thus it is only required to approximate $\operatorname{MSE}\left(\hat{a}\left(k, t_{0}\right)\right)=\operatorname{MSE}_{k}\left(\hat{\boldsymbol{B}}\left(t_{0}\right)\right)$ by the approximated bias and variance.

To find the pilot bandwidth $h^{*}$, the intersection of confidence intervals (ICI) method is adopted. The ICI method is an empirical adaptive bandwidth selection method proposed by Goldenshluger and Nemirovski [13], and it has been successfully applied to various areas, including local polynomial regression, image processing, and time-frequency analysis, for selecting the variable bandwidth [11]-[12]. The algorithm of ICI is omitted to save space, and more details can be found in [11]-[12].

Suppose we have used the ICI method to obtain the variable bandwidths $h_{I C I}\left(k, t_{0}\right)$ for the $k$-th coefficient at each time instant $t_{0}$. Then, $h_{I C I}\left(k, t_{0}\right)$ will be used as pilot bandwidths $h^{*}\left(k, t_{0}\right)$ to compute $X^{*}, W^{*}$, and $\hat{\beta}^{(p+v)}\left(k, t_{0}\right)$ in (11)-(13). Next, with the pilot estimates, we can calculate a series of bias, variance, and MSE values of the LPM estimators using each bandwidth in the set $\boldsymbol{H}$. Finally, the optimal data-driven bandwidth is the bandwidth having the minimum MSE, i.e.,

$$
h_{M M S E}(k, t)=\arg \min _{h} \operatorname{MSE}\left(\hat{a}\left(k, t_{0}\right)\right) .
$$

\section{ONLINE IMPLEMENTATION AND PRACTICAL ISSUES}

The LPM is originally proposed in [13] with the support of two-side kernels so that both past measuremrents and future measurements can be used. However, in many practical applications, such as the estiamting of voltage flicker in power systems, only past measauremrents are avaiable and thus online tracking algorithm of the LPM is desirable. Actually, the LPM method can be easily extended for the purpose of online tracking by employing a one-sided kernel. For example, the one-sided Epanechnikov kernel is given by:

$$
K(u)=\left\{\begin{array}{cc}
\frac{3}{4}\left(1-u^{2}\right) & -1<u \leq 0 \\
0 & u \leq-1 .
\end{array}\right.
$$


The above one-sided Epanechnikov kernel has a support of $\left(t_{0}-h, t_{0}\right)$, so its effective length is $h$.

As observed in [10] and [13], the varying optimal bandwidth $h_{M M S E}(k, t)$ usually exhibits considerable variability since it is based on a finite sample approximation. Hence, to obtain a smooth bandwidth function, the optimal variable bandwidth $h_{M M S E}(k, t)$ can be further smoothed recursively to obtain the smoothed optimal bandwidth function $\widetilde{h}_{\text {opt }}(k, t)$ as

$$
\tilde{h}_{\text {opt }}(k, t)=\lambda_{h} h_{M M S E}(k, t)+\left(1-\lambda_{h}\right) M_{h},
$$

where $\lambda_{h} \in(0,1)$ is the forgetting factor, which should be close to 1 to ensure the smoothness of the resultant bandwidth function, $M E D_{h}=\operatorname{median}\left(h_{M M S E}(k, t-1), \cdots, h_{\text {MMSE }}\left(k, t-I_{l}\right)\right)$ and $I_{l}$ is the length of smoothing window. The use of the median operation avoids possible large variations in $h_{M M S E}(k, t)$ from affecting significantly the smoothed bandwidth $\tilde{h}_{\text {opt }}(k, t)$.

The proposed LPM with variable bandwidth selection (LPM-VBS) method is summarized as follows.

Step 1. For each bandwidth $h$ in $\boldsymbol{H}$ of (10), an estimate of the coefficient $\hat{a}(k, t ; h)$ is calculated by a $p$-th order LPM with the constant bandwidth $h$ at every time instant using (7).

Step 2. A pilot bandwidth $h_{I C I}(k, t)$ is estimated using the ICI method based on the estimates $\hat{a}(k, t ; h)$.

Step 3. For each bandwidth $h$ in $\boldsymbol{H}$, a $\left(p+p_{e x}\right)$-th order LPM with the pilot bandwidth $h_{I C I}(k, t)$ is carried out to approximate the $\operatorname{MSE} \operatorname{MSE}(\hat{a}(k, t ; h))$ using (11)-(14).

Step 4. At each time instant, the optimal bandwidth $h_{M M S E}(k, t)$ is approximated as the bandwidth having the minimum MSE (15), and the final smoothed variable bandwidth $\tilde{h}_{\text {opt }}(k, t)$ is obtained recursively as (17).

Step 5. A $p$-th order LPM with $\tilde{h}_{\text {opt }}(k, t)$ is finally performed to obtain the final estimates of the time-varying coefficient $\hat{a}\left(k, t ; \tilde{h}_{o p t}(k, t)\right)$.

We now discuss the parameter selection of the online LPMVBS method. Firstly, to determine the bandwidth set $\boldsymbol{H}$ of (7), the smallest bandwidth value should be selected as the minimum bandwidth that makes (7) solvable (overdetermined). Because a $\left.\left(p+p_{e x}\right)\right)$-th order LPM is used to approximate MSE, the number of samples included in the one-sided kernel should be larger than $\left(p+p_{e x}+1\right) L$ (the dimension of $\hat{\boldsymbol{\beta}}(t))$. If the data are uniformly distributed at a sampling frequency of $f_{s}$, it is required that

$$
h_{0} \geq\left(p+p_{e x}+1\right) L / f_{s} .
$$

Moreover, to achieve a good tradeoff between performance and complexity, in our study, the largest bandwidth of $\boldsymbol{H}$ is selected to make the largest kernel cover at least $1 / 8$ of the data set, and the number of bandwidths, $J$, is set as 4 .

As for the selection of polynomial order $p$ and $p_{e x}$, although a high order might lead to a smaller bias in theory, it does not imply that the polynomial order can be arbitrarily large due to two reasons. First, $\left(p+p_{e x}+1\right) L$ should be smaller than the number of measurements in the smallest kernel to ensure that the LPM solution in (7) is well-posed. Secondly, a higher order increases the variability of the LPM estimator because it implies more parameters (with the number $\left.\left(p+p_{e x}+1\right) L\right)$ to be estimated. More details about the influence of polynomial order on the variance and the selection of $p$ and $p_{e x}$ can be found in the comprehensive book by Fan and Gijbels [10]. They also recommended a local linear estimator $(p=1)$ because, compared to local constant estimator $(p=0)$, the linear estimator will not suffer from any loss in variance and can reduce the bias effectively. Therefore, in this paper, the polynomial order is chosen as $p=1$. The excess order $p_{e x}$ is generally chosen as 2 , as recommended in [10]. The proposed bandwidth setting gave satisfactory results in our experiments.

\section{SiMULATION RESULTS}

\section{A. Random-coefficient Linear Models}

We first test the performance of the proposed LPM-VBS method in a random-coefficient linear model under different noise situations. In the stimulated random-coefficient linear models, the time-varying coefficients were generated by filtering white Gaussian signals with zero mean and unit variance using low-pass filters. The resultant TVLM has a coefficient function varying considerably over time and the extent of variations is determined by the cutoff frequency of the low-pass filter. Four cutoff frequencies $f_{c}$ (normalized by the sampling rate $f_{s}=512 \mathrm{~Hz}$ ): $0.01,0.02,0.05,0.1$, were used to simulate different extents of coefficient variations. Zero mean white Gaussian noises with different SNRs: 20, 10, 5, and $0 \mathrm{~dB}$, were added to simulate different noise conditions. The order of the TVLM was $L=4$. The sampling rate was $512 \mathrm{~Hz}$, and the number of measurements was $n=512$. The polynomial order used in LPM was $p=1$ and $p_{e x}=2$. The LPM-VBS algorithms with one-sided Epanechnikov kernels and two-sided Epanechnikov kernels are both tested. For LPM using two-sided kernels, $\boldsymbol{H}=\{1 / 64,1 / 32,1 / 16,1 / 8\}$, while for LPM using one-sided kernels, $\boldsymbol{H}=\{1 / 32,1 / 16,1 / 8,1 / 4\}$.

Here, we compare the proposed LPM-VBS method with other conventional TVLM identification methods, including the RLS, the KF, and the BEM methods. For quantitative comparison, the ensemble mean squared deviation (EMSD) from the true coefficients was calculated and used as the performance measure in the whole time period:

$$
E M S D=10 \log _{10}\left\{\frac{1}{n} \sum_{i=1}^{n} \sum_{k=1}^{L}\left[a\left(k, t_{i}\right)-\hat{a}\left(k, t_{i}\right)\right]^{2}\right\} .
$$

In BEM, polynomial basis was employed and a set of expansion orders, $p_{B E M}=[1,5,10,15,20]$, was tested to 

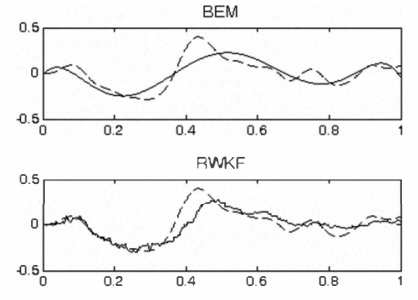

LPM-VBS with $h_{C,}$ (one-sided)

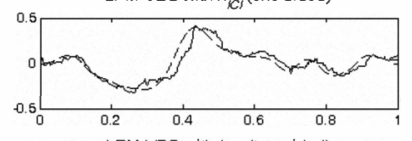

LPM-VBS with $h_{\text {ICI }}$ (two-sided)

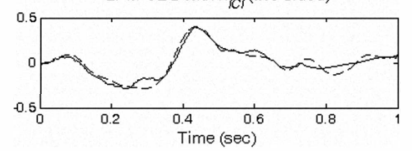

Fig. 1. Comparisons between various TVLM identification methods for random-coefficient linear systems $\left(f_{c}=0.02 \mathrm{~Hz}, \mathrm{SNR}=5 \mathrm{~dB}\right)$. Dash lines denote the true coefficients and solid lines are the estimates.
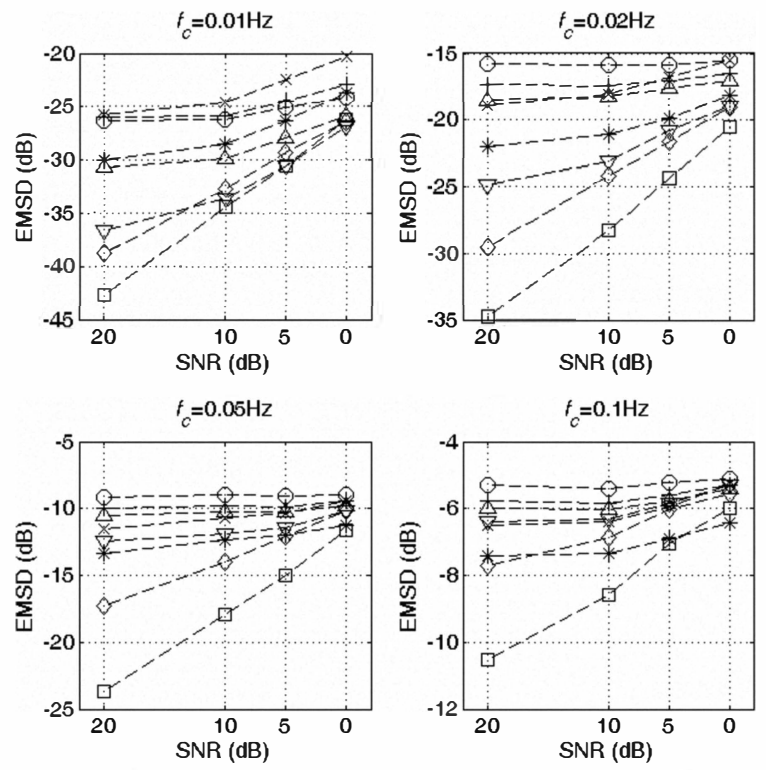

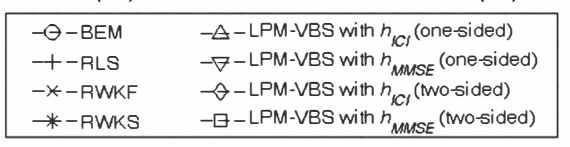

Fig. 2. EMSD comparisons between various TVLM identification methods for random-coefficient linear systems.

determine the one with the best EMSD for comparison with other TVLM methods. For RLS, a set of forgetting factors, $\lambda=[0.8,0.85,0.9,0.95,0.99]$, was tested, and the results with the best EMSD were selected for further comparison. The random walker KF (RWKF), where the state transition matrix was chosen as an identity matrix, is tested for comparison, and the covariance matrices of state noise and observation noise were estimated recursively using the algorithms in [16]. A fixed-interval random walk Kalman smoother (RWKS) is also tested because it can take advantage of future measurements to effectively avoid the tracking lag problem of the RWKF.

Fig. 1 shows the estimation results of one coefficient of an example four-order TVLM $\left(f_{c}=0.02 \mathrm{~Hz}\right.$ and SNR $\left.=5 \mathrm{~dB}\right)$, which
TABLE I. EMSD COMPARISONS BETWEEN VARIOUS TVLM IDENTIFICATION METHODS FOR RANDOM-COEFFICIENT LINEAR SYSTEMS (UNIT: dB)

\begin{tabular}{|c|c|c|c|c|}
\hline \multirow{2}{*}{ Methods } & \multicolumn{4}{|c|}{$f_{c}=0.01 \mathrm{~Hz}$} \\
\hline & $\mathrm{SNR}=20$ & $\mathrm{SNR}=10$ & $\mathrm{SNR}=5$ & $\mathrm{SNR}=0$ \\
\hline BEM & -26.36 & -26.18 & -25.12 & -24.14 \\
\hline RLS & -25.98 & -25.89 & -24.55 & -22.86 \\
\hline RWKF & -25.75 & -24.64 & -22.48 & -20.27 \\
\hline RWKS & -30.01 & -28.50 & -26.32 & -23.71 \\
\hline LPM-VBS ${ }^{(1)}$ with $h_{I C I}$ & -30.62 & -29.93 & -27.99 & -25.93 \\
\hline LPM-VBS $^{(1)}$ with $h_{M M S E}$ & -36.56 & -33.68 & -30.55 & -26.85 \\
\hline LPM-VBS ${ }^{(1)}$ with $h_{I C l}$ & -38.72 & -32.68 & -29.31 & -26.51 \\
\hline LPM-VBS $^{(\text {II) }}$ with $h_{\text {MMSE }}$ & -42.66 & -34.49 & -30.60 & -26.21 \\
\hline \multirow{2}{*}{ Methods } & \multicolumn{4}{|c|}{$f_{c}=0.02 \mathrm{~Hz}$} \\
\hline & $\mathrm{SNR}=20$ & $\mathrm{SNR}=10$ & $\mathrm{SNR}=5$ & $\mathrm{SNR}=0$ \\
\hline BEM & -15.80 & -15.91 & -15.86 & -15.53 \\
\hline RLS & -17.37 & -17.43 & -17.01 & -16.51 \\
\hline RWKF & -18.85 & -18.02 & -16.80 & -15.49 \\
\hline RWKS & -22.00 & -21.08 & -19.89 & -18.16 \\
\hline LPM-VBS $^{(\mathrm{l})}$ with $h_{\mathrm{ICI}}$ & -18.48 & -18.23 & -17.60 & -17.03 \\
\hline LPM-VBS ${ }^{(1)}$ with $h_{M M S E}$ & -24.91 & -23.05 & -20.84 & -18.96 \\
\hline LPM-VBS ${ }^{(1 I)}$ with $h_{I C l}$ & -29.49 & -24.15 & -21.64 & -19.10 \\
\hline LPM-VBS $^{(\text {(II) }}$ with $h_{\text {MMSE }}$ & -34.79 & -28.33 & -24.33 & -20.55 \\
\hline \multirow{2}{*}{ Methods } & \multicolumn{4}{|c|}{$f_{c}=0.05 \mathrm{~Hz}$} \\
\hline & $\mathrm{SNR}=20$ & $\mathrm{SNR}=10$ & $\mathrm{SNR}=5$ & $\mathrm{SNR}=0$ \\
\hline BEM & -9.19 & -8.98 & -9.07 & -8.93 \\
\hline RLS & -10.01 & -9.83 & -9.86 & -9.41 \\
\hline RWKF & -11.49 & -10.72 & -10.38 & -9.47 \\
\hline RWKS & -13.34 & -12.32 & -12.02 & -11.25 \\
\hline LPM-VBS $^{(1)}$ with $h_{I C l}$ & -10.56 & -10.29 & -10.24 & -9.83 \\
\hline LPM-VBS $^{(1)}$ with $h_{M M S E}$ & -12.45 & -11.89 & -11.40 & -10.19 \\
\hline LPM-VBS ${ }^{(\mathrm{II})}$ with $h_{I C l}$ & -17.31 & -13.98 & -12.05 & -10.19 \\
\hline LPM-VBS $^{(\text {(I) }}$ with $h_{\text {MMSE }}$ & -23.67 & -17.91 & -14.98 & -11.65 \\
\hline \multirow{2}{*}{ Methods } & \multicolumn{4}{|c|}{$f_{c}=0.1 \mathrm{~Hz}$} \\
\hline & $\mathrm{SNR}=20$ & $\mathrm{SNR}=10$ & $\mathrm{SNR}=5$ & $\mathrm{SNR}=0$ \\
\hline$\overline{\mathrm{BEM}}$ & -5.31 & -5.40 & -5.22 & -5.12 \\
\hline RLS & -5.77 & -5.84 & -5.60 & -5.30 \\
\hline RWKF & -6.50 & -6.39 & -5.94 & -5.25 \\
\hline RWKS & -7.41 & -7.33 & -6.89 & -6.43 \\
\hline LPM-VBS $^{(\mathrm{l})}$ with $h_{I C I}$ & -5.98 & -6.03 & -5.76 & -5.40 \\
\hline LPM-VBS $^{(1)}$ with $h_{M M S E}$ & -6.38 & -6.32 & -5.84 & -5.29 \\
\hline LPM-VBS ${ }^{(\text {II) }}$ with $h_{\mathrm{Cl}}$ & -7.71 & -6.85 & -6.01 & -5.57 \\
\hline LPM-VBS $^{(\text {(I) }}$ with $h_{M M S E}$ & -10.50 & -8.59 & -7.07 & -5.99 \\
\hline
\end{tabular}

(I) One-sided kernel; ${ }^{\text {(II) }}$ two-sided kernel.

has both slowly changing and rapid changing coefficients. It can be seen clearly that, BEM gives a very smooth estimation, but rapid change around 0.4 second is smoothed out. The RLS, RWKF, and RWKF have better tracking abilities than BEM, but it is still difficult for them to track rapid changes accurately. Overall, the LPM-VBS methods are better than other methods in this example and they have a good adaptability for both rapid changes and slow-varying parts.

Table I listed the EMSD values averaged over 100 independent runs, and these values were presented graphically in Fig. 2. We can conclude that, 1) the LPM-VBS method has better performance than other methods for TVLM's having various degrees of coefficient variations and different noise levels; 2) the LPM using one-sided kernel has relatively lower EMSD values than other tracking methods when the noise is small and the variability of the system is not large, but the performances of all methods are comparable under heavy noise and large system variability; 3) RWKS had a better result than LPM when the SNR is small and coefficient variation is large. In conclusion, the LPM method, which is more data-dependent, has an evident advantage over $\mathrm{BEM} / \mathrm{RWKF} / \mathrm{RWKS}$, which is more model-dependent, when a prior model is not precisely given and the SNR is large. 


\section{B. Tracking of Voltage Flicker in Power Systems}

Lastly, we evaluate the performance of the proposed LPMVBS method in tracking the envelope of the flicker and compare it with conventional RLS and RWKF methods [5]. The measured voltage is assumed to be

$$
v(t)=\left\{\begin{array}{cc}
(2+\cos 20 \pi t) \cos (100 \pi t+\pi / 3) & 0<t \leq 0.15 s, \\
(4+\cos 20 \pi t) \cos (100 \pi t+\pi / 3) & t>0.15 s .
\end{array}\right.
$$

The envelope of the voltage, which was modulated by a sinusoid, fluctuated periodically and mildly, while a rapid change occurred at time $0.15 \mathrm{~s}$. The sampling rate was set at $1600 \mathrm{~Hz}$ (32 samples per cycle). A zero mean white Gaussian noise component with $\mathrm{SNR}=30 \mathrm{~dB}$ was added.

In the RLS algorithm, the forgetting factor was 0.7 , as recommended in [5]. In the RWKF method, the state transition matrix and state noise matrix were set to identity matrices, and the covariance matrices of state and observation noises were estimated recursively as in [16]. For tracking purpose, LPMVBS with one-sided kernel was tested, and the bandwidth set was $\boldsymbol{H}=\{1 / 200,1 / 100,1 / 50,1 / 25\}$. Other parameters for the LPM were the same as those in previous simulations.

We can see from Fig. 3 that the proposed LPM-VBS method achieved a good envelope tracking performance for both rapid voltage change and slow voltage fluctuations. On the other hand, the RLS and RWKF methods showed a slow response to rapid change, with the RLS showing a slow convergence rate. By comparing the EMSD values of the three methods (RLS: $-4.13 \mathrm{~dB}$, RWKF: $-11.61 \mathrm{~dB}$, LPM-VBS: $-16.79 \mathrm{~dB}$ ), we can conclude that the LPM-VBS had a better envelope tracking performance than RLS and RWKF.

\section{CONCLUSION}

A local polynomial modelling method for identification of time-varying linear models and its application to estimate voltage flicker in power systems were presented in this paper. Simulation results showed that the performance of the LPM with variable bandwidth selection method was superior to several conventional TVLM identification methods in an example of voltage envelope tracking. The LPM method is expected to find various other applications like change detection and diagnosis in power systems and delivery.

\section{REFERENCES}

[1] A. A. Girgis, J.W. Stephens, and E. B. Makram, "Measurement and prediction of voltage flicker magnitude and frequency," IEEE Trans. Power Delivery, vol. 10, pp. 1600-1605, July 1995.

[2] P. K. Dash, D. P. Swain, A. C. Liew, and S. Rahman, "An adaptive linear combiner for on-line tracking of power system harmonics," IEEE Trans.Power Syst., vol. 11, pp. 1730-1735, Nov. 1996.

[3] T. Zhang and E. B. Makram, "Wavelet representation of voltage flicker," Elect. Power Syst. Res., vol. 48, pp. 133-140, 1998.

[4] S. A. Soliman and M. E. El-Hawary, "Measurements of power system voltage and flicker levels for power quality analysis: A static LAV state estimation based algorithm," Int. J. Elect. Power Energy Syst., vol. 22, pp. 447-450, 2000.

[5] M. I. Marei, E. F. El-Saadany, and M. M. A. Salama, "Envelope tracking techniques for flicker mitigation and voltage regulation," IEEE Trans. Power Deliv., vol. 19, no.4, pp. 1854-1861, Oct., 2004. (a)

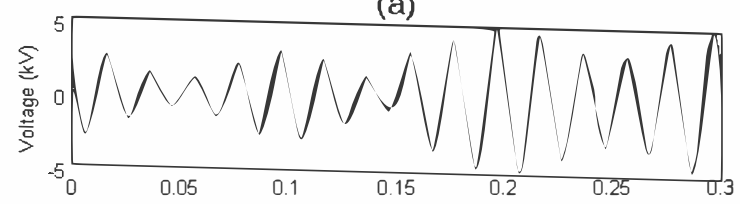

(b)

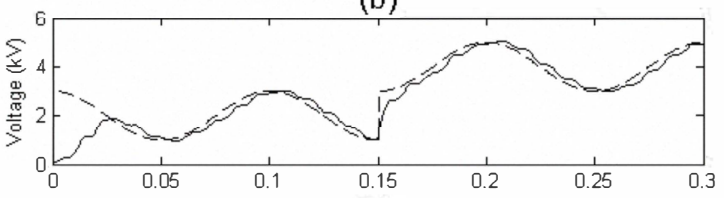

(c)

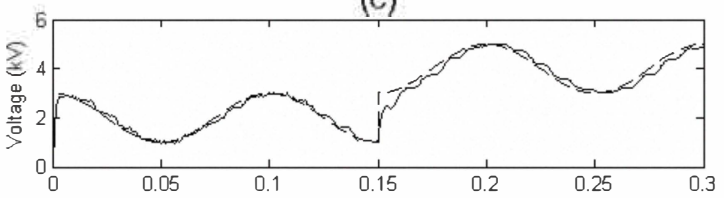

(d)

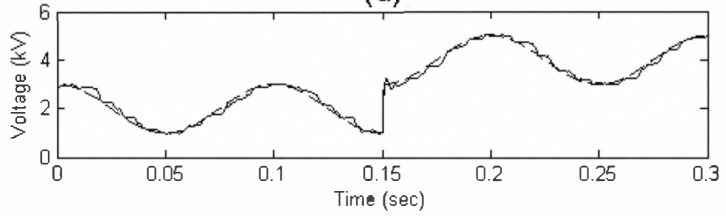

Fig. 3. Comparisons between various methods for tracking envelope of voltage filcker: (a) the measured voltage, (b) RLS, (c) RWKF, (d) LPMVBS. Dash lines in (b)-(d) denote the true envelope and solid lines are the estimates.

[6] M. Niedzwiecki, Identification of Time-varying Processes, Chechester: John Wiley, 2000.

[7] J. Proakis, C. Rader, F. Ling, C. Nikias, M. Moonen, and I. Proudler, Algorithms for Statistical Signal Processing, Englewood Cliffs, NJ: Prentice Hall, 2002.

[8] C. K. Chui and G. Chen, Kalman Filtering: with Real-time Applications, 2nd ed. Berlin; New York: Springer, 1999.

[9] T. S. Rao, "The fitting of non-stationary time-series models with timedependent parameters," J. R. Stat. Soc. Ser. B, vol. 32, no. 2, pp.312$322,1970$.

[10] J. Fan and I. Gijbels, Local Polynomial Modelling and Its Applications, London: Chapman and Hall, 1996.

[11] V. Katkovnik, K. Egiazarian, and J. Astola, Local Approximation Techniques in Signal and Image Processing, Bellingham: SPIE Press, Sept. 2006.

[12] Z. G. Zhang, S. C. Chan, K. L. Ho, and K. C. Ho, "On bandwidth selection in local polynomial regression analysis and its application to multi-resolution analysis of non-uniform data," J. Signal Process. Syst., vol. 52, no. 3, pp. 263-280, Sept. 2008.

[13] S. C. Chan and Z. G. Zhang, "Local polynomial modelling and bandwidth selection for time-varying linear models," in Proc. International Conferences on Information, Communications and Signal Processing (ICICS 2009), Macau, China, 7-10 Dec., 2009.

[14] Z. G. Zhang, S. C. Chan, and Y. S. Hung, "Local polynomial modelling of time-varying autoregressive processes and its application to the analysis of event-related electroencephalogram," in Proc. IEEE International Symposium on Circuits and Systems (ISCAS2010), Paris, France, 30 May-2 June, 2010.

[15] A. Goldenshluger and A. Nemirovski, "On spatial adaptive estimation of nonparametric regression," Math. Methods Statist., vol. 6, no. 2, pp. 135-170, 1997.

[16] Z. G. Zhang, S. C. Chan, and K. M. Tsui, "A recursive frequency estimator using linear prediction and a Kalman filter-based iterative algorithm," IEEE Trans. Circuits Syst. II, vol. 55, no. 6, pp. 576-580, June 2008. 